\title{
Analysis and simulation of the adhesion forces between clot and the artery wall for a novel thrombectomy device applied to the Middle Cerebral Artery.
}

\author{
G. Romero, I. Higuera, M.L. Martinez \\ ETSI Engineering, Universidad Politecnica de Madrid \\ Madrid, Spain \\ e-1nail: gregorio.ronero (a) upm.es, \\ irene.higuera $(\bar{a}$ upm.es, luisa.mtzmuneta $(\hat{a})$ upm.es
}

\author{
G. Pearce, N.D. Perkinson \\ Wolverhampton University \\ Wolverhapton, United Kingdom \\ e-mail: gillpearce $(\bar{a}$ googlemail.com, \\ neil(a)perkinson.org.uk
}

\begin{abstract}
A number of Thrombectomy devices using a variety of methods have now been developed to facilitate clot removal. We present research involving one such experimental device recently developed in the $\mathbf{U K}$, called a 'GP' Thrombus Aspiration Device (TAD). This device has the potential to improve the process of extracting thrombosis clots in the cerebral arteries. The development of a simulation model for this device is shown using Bond-Graph formalism applied to modeling and simulating the adhesion forces between the clot and the artery wall. Bond-Graph technique is a visual methodology that adds more Uransparency to the processes and it has turned out to be remarkably useful as it is a simple, effective method that can be applied to any physical system where there is a power exchange.

Such modelling appears to be able to simulate the device under a variety of conditions which may assist in the optimisation of the ' $G P$ ' TAD device if the novel design features of the device can be effectively incorporated into the model.
\end{abstract}

Keyw'ords- Biomedical engineering, Thrombectomy Device, Adhesion forces, Bond Graph technique.

\section{INTRODUCTION}

Stroke is a major cause of morbidity and montality worldwide. In the UK there are 130,000 strokes each year [1]. Even patients who survive the initial insult of such a cerebral vascular event are often left with residual disability resulting in profound impact on their life and life expectancy. Approximately $85 \%$ of strokes are caused by a blood clot. Imravenous thrombolysis using alteplase is an effective treatment for acute stroke [2]. However, there are limitations on its use. Due to the risk of hemorluage it is coniraindicated in patiens who have undergone recem surgery. In addition, it has to be given at a relatively early time after the siroke las occurred and it is only currently licensed for use up to 3 hours post stroke [3]. A more recent study has indicated that it could possibly be used up to 4 to 5 hours post-stroke. It can therefore only currently be nsed in particular patients who have suffered a stroke.

During the last decade Thrombectomy Devices have become inore widely used and have been developed as an altemative means for clot removal. The need to study new
Thrombectomy devices like the one described here means that a computer pre-modelling may be helpfulin the optinization and fine-tuning of such devices prior to clinical trials. The extraction of the obstnicting element (i.e. the blood clot) by the ' $\mathrm{GP}$ ' $\mathrm{TAD}$ is done by means of vacuum suction provided by the action of a pump represented in the model as an effor source. To do this, an increasing pressure will be initially considered over time until it takes on some values that are ideal for generating the suction required to remove the clot and which are suited to this type of operation. The force required to separate the clot from the arterial wall will be analysed by modelling a complianceresistance system to represent the interaction between both parts. A viscous damper will be used that allows considering the change from static to dynamic friction in its relative movement. To implement the phenomena by defining the different sections of the mechanism studied in the paper. blood is deemed to be an incompressible fluid. while the tube between the pump and the 'GP' device is deemed to be nonelastic. The loss load will be due mainly to the existent flow, which by means of previous studies las been shown to be laininar.

The main objective of the development of this simulation model is to analyze the minimum pressure required to perfonn the extraction according with different adhesion forces between clot and the arlery wall. and to check that, both the pressure and the time required to complete the clot extraction are reasonable for use in clinical situations, and are consistent with any experimentally obtained data. In addition, it nust be checked that that the cerebral arteries are able to support the force to which they are subjected, as well as the components comprising the device, and that the blood flow generated acquires speeds that fall within admissible parameters. These studies and confimations will allow optimising the device to ensure its possible future use in patiens with thrombosis.

This paper gives a brief description of the device under study, as well as the parts comprising it. Next the model used for the simulation is described and the phenomena considered to define the device, and, in addition, the values of the parameters nsed are defined. Lastly the results obtained and the conclusions of this study are attached. 


\section{II. 'GP' TAD STRUCTURE}

The 'GP' device (figure 1) [4] action involves using a vacuum pump that provides the necessary suction pressure for the operation, joined to a very long catheter; the 'GP' device is located at the end of this catheter. The proposed procedure for using this device is to introduce it into an antery in close proximity to the occluding blood clot, and to position it at a distance of approximately $3 \mathrm{~mm}$ from the clot. Then the suction would begin until the clot is extracted. The clot would crosss the $3 \mathrm{~mm}$ that separates it from the 'GP' device and clot capture would occur and the device would be removed from the body.

It is cunrently being developed as a potential thrombus aspiration device through a series of in vitro studies. This device has the potential to be used in relatively small arteries. It has no moving parts and therefore should reduce the risk of breakage in a vessel. Since it does not tonch the clot itself it also has the potential to reduœ the risk of clot disruption and downstream embolisation. Thrombectony is achieved by aspiration through a catheter in which the 'GP' device is embedded. The intemal surface has been mathematically designed. It is also associated with low forces at the periphery of the device which may therefore reduce the risk of arterial collapse during aspiration of the $\operatorname{clot}[5,6,7]$.

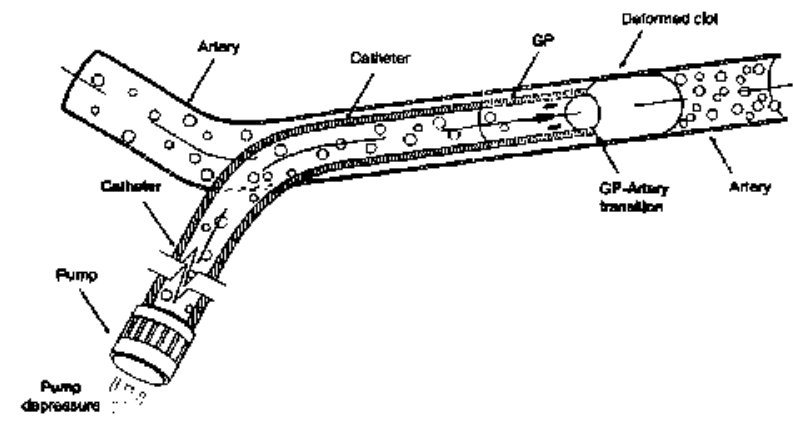

Figure 1. Schematic view of the GP Thrombus Aspiration Device.

The 'GP' Thrombns Aspiration Device (TAD) has been modelled using a 3-D FLUENT $\mathrm{C}$ simulation software package [8]. In addition, different existing techniques have been considered for the modelling and simulation [9]. Some methods which have been considered for their applicability are the Boltzmann flow simulation technique. and finite elements modelling and its implementation in Matlab (C) software, with 2D or 3D models; or by means of Laplace uransformations using Dynamic Motion Solver $(\mathcal{C}$ software.

The objective of this study is to imlroduce a different method to reproduce the model and it can be used in showing the potential perfonmance of the 'GP' TAD device under different conditions of blood flow, size of blood clot, in a given vessel. The method chosen for the representation and simulation of uis model is the Bond Graph technique [10, $11,12]$, which allows assimilating the nodel to an electric circuit made up of resistances, capacitances and inductances. Therefore. it is possible to obtain the results in a simple way by evaluating flows and efforts that join and connect the conponents of the model.

\section{Modelling THE 'GP' TAD DEvice}

A pump creates the necessary pressure to cany out the extraction, and is positioned at the other end of the catheter that does not contain the 'GP' TAD. In the model, it can be represented by a variable pressure source whose value will increase from zero to a non-detennined value $(0-[-30,-60]$ kPa) suitable for carrying out the operation of clot removal and will be obtained from the optinization of the developed model. The time taken to reach the maximum value of pressure has been obtained from expeniment and is about 2-3 sec., after which time the pressure provided by the pump remains constant.

To generate the correct model, we need assimilate that the catheter, 'GP' device and artery as a pipe of different materials, in which it is necessary to analyze the inertances due to mass of fluid, the compressibility that the blood and artery are subjected to, and finally the resistances that appear when fluid and blood clot flow into the pipe [13]

\section{A. Inertances}

The flow inertia to be overcome in the movement from clot to pump can be modelled with the flow and the section. Considering diameters (circular geometry) and lenguhs of the different sections (catheter, the GP device or the space between the GPTAD and clot), we can assume the blood inerance with the following expression:

$$
I=\frac{\rho \cdot L}{\pi \cdot(D / 2)^{2}}
$$

In addition to the previous inertance inserted, the model must lave an inertance that represents the mass of the clot and it will be defined in next section IV.

\section{B. Compresibilities}

The blood acts as a compliance producing a decrease in volume when the pressure required for conpression is increased and it is necessary take account of the blood compressibility. This belhaviour is dependent on the Bulk's blood coefficient and section of the artery. If we assume a circular geometry. it can be defined as a capacilance of $K$ value:

$$
K=\mathbf{4} \cdot B / \pi \cdot D_{a}^{2} \cdot L
$$

In addition, we can assume that catheter and 'GP' device are incompressible due to the high rigidity, but it is necessary to inser the compressibility of the length of artery that appears between the GP device and the clot, in line with its Young's modulus:

$$
K=\frac{E \cdot h}{V_{0} \cdot 2 \cdot r_{0}}
$$

where ' $V_{0}$ ' is the artery initial volume and ' $r_{0}$ ' is the artery initial radius. 


\section{Resistances}

Linear load loss is due to the friction between the liquid particles and the anery walls. To simplify. only linear load losses are taken into account. If we suppose that the catheter is composed of successive horizomal pipes of constant cross section in each section. the load loss is reduced to a pressure loss as the fluid advances along the catheter, the loss being progressive and proportional to the length of the catheter. If we assume that the blood flow is laninar due to the Reynolds number being approximately $1.000(\operatorname{Re}<2.200)$, the equation that governs the behaviour in the catheter can be detenmined by the following expression:

$$
R=\frac{128 \eta \cdot L}{\pi \cdot D^{4}}
$$

In order to calculate the effect of the entire catheter in this model. (due to the lenguh). it can be partitioned in identical sections (ten sections) and was represented by ten sub-models that include the previous described parameters.

Some studies show that in diseased anteries viscosity bloodstream varies, increasing its value but for this first approximation we used a normal value of $0,0035 \mathrm{~Pa} \cdot \mathrm{s}$.

Due to the 'GP' TAD device (see figure 1) is positioned near the clot, the resistance of the blood flow can be represented by the same eq. (1) with the corresponding values.

In addition, the antery being located near the end of the 'GP' TAD and it is necessary to consider the transition between both elements as a secondary load loss caused by the deflecting nozzle due to the difference in diameter of the 'GP' device and the arery respectively and the subsequent vanations in flow. These load losses can be represemed as a resistance and can be calculated as a fumction of the mean value $\left(\mathrm{D}_{\mathrm{m}}\right)$ between the diameter of the catheter and the diameter of the artery, and the load loss coefficient $(\xi)$ which is a dimensionless parameter that quantifies the loss produced and depends on the geometry of the deflecting nozzle, with the following expression:

$$
R=8 \cdot \rho \cdot \xi \frac{Q}{\pi^{2} \cdot D_{m}{ }^{4}}
$$

where ' $Q$ ' is the flow' which circulates in the section between the end of the 'GP' device and the artery.

Once all this was defined in the model, it was necessary to change from the hydraulics to mechanics domain before considering the clot element. to be able to evaluate the clot movement and effon involved in clot extraction taking imo account the physical friction between the clot and the artery.

To formulate the corresponding equations from the differem described phenomena, it was necessary to assume that the flow after and before resistances and inertances are the same; neverheless, with this assumption in respect of after and before compliances the pressures are similar.

\section{MODELLING THE ADHESION FORCES BETWEEN CLOT AND THE ARTERY.}

Accurately defining the clot model in order to model it is the most complex part of the model. A clot is a cylindricallyshaped elemem of [3-5] $\mathrm{cm}$ long, and of a mass that fails between [0.5-1] gram. This element has usually formed in another location usually in a vascular anery, and has become dislodged remaining trapped in smaller diameter anteries. such as the cerebral artery considered in this work. Therefore, due to this difference in diameter, the clot becomes attached to the wall by a force that needs to be overcome in order to begin its movement for removal. In addition the relative movement between the clot and the antery presents static and dynamic friction, which needs to be taken into account. If a correct approximation to reality is to be achieved all these phenomena, as well as the circumstances restricting clot movemem, need to be considered.

As already stated the phenomenon preventing clot movemem is the difference in diameter between the clot and the anery where it is located. Experimental data demonsurated that low times of residence of the clot in the antery, the value of the adhesion force is $0,01 \mathrm{~N}$; and with higher times. this value grows up to $0 . \mathrm{IN}$. In this paper three different forces are going to be compared: $0,1 \mathrm{~N}, 0,01 \mathrm{~N}$ and $0,001 \mathrm{~N}$.

In order to decide when to begin the movement of the obstnictive element. firstly we need to iusen a spring into the model to measure the force supported by the beginning of the clot and its defonmation.

To obtain the value of this spring the phenomenon of surface tension nust be taken imo account, since it is this that joins the clot to the arery. This suface teusion $\gamma$ comes about from the attraction forces between molecules and is defined as force by unit length.

$$
\gamma=\frac{F}{l}
$$

If we take the sphere in figure 2 , the surface tension would act on the circumference of the contact between the clot and the anery (marked with a blue ellipse).
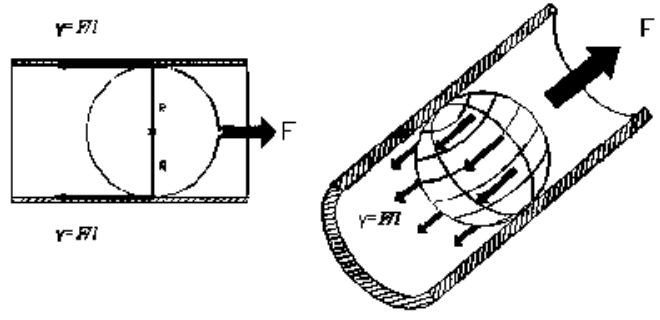

Figure 2. Surface tension.

Bearing in mind the above. if we take into accoum the value of the length of contact from the radius $r$ of the antery, the value for the surface tension can be obtained and, in tum, the value for the spring coefficient as: 


$$
K=\gamma=F / l=1,061 \mathrm{~N} / \mathrm{m}
$$

As stated above, the clot is between [3-5] $\mathrm{cm}$ long, which means it can be broken down into the union of several spheres, all with the same constant. Since over the whole surface of the clot there are adhesion forces, to obtain a correct approximation it is necessary to consider the existence of a sphere for every $0,1 \mathrm{~mm}$. This means that between 300 and 500 spheres located in parallel, with each sphere in contact at 2 diametrical opposite points with the artery wall, would need to be included in the model. On the other hand, since the clot behaves like a rigid body, the fact that all the spheres are located in parallel must be taken into account, so that until the resultant adhesion force is overcome in all of the spheres, the clot will not begin to move.

Therefore, since all the individual springs are equal, the equivalent spring for a $5 \mathrm{~cm}$ clot can be had from the form appearing in the following expression:

$$
K_{e q}=\frac{K}{n}=\frac{1,061}{500}=0,002122 \mathrm{~N} / \mathrm{m}
$$

To know when the force will be reached in this equivalent spring and, there-fore, when the clot movement will begin, it is essential to calculate the displacement of the spring when it is subjected to $0,01 \mathrm{~N}$ (i.e.) through a typical spring equation. Therefore, only when the spring undergoes this displacement should the clot be allowed to move; to the contrary it would be prevented.

$$
x=\frac{F}{K_{e q}}=\frac{0,01 N}{0,002122 \mathrm{~N} / \mathrm{m}}=4,7124 \mathrm{~m}
$$

Secondly, the friction between the clot and the arterial wall creates another resistance factor. The value of this parameter must be variable depending on whether the clot has not begun its movement (static friction) or if it is already in movement (dynamic friction), so that when the clot begins to move the friction value will drop considerably. This value is obtained starting from the Stokes equation and can be given a value of $2,5 \cdot 10^{-6} \mathrm{~N} \cdot \mathrm{s} / \mathrm{m}$ for the static friction and an order of magnitude lower than for the dynamic friction.

The transition between both values marks the beginning or end of the clot movement by means of the displacement of the spring representing the deformation described in expression (9). So, when the displacement undergone by this spring is less than that calculated, static friction will rule; to the contrary, if it is greater, the friction will be dynamic.

In addition to the spring and the resistance inserted, the model must have an inertance element that represents the mass of the clot.

Finally, to ensure that the clot remains at rest while the force existing at its beginning is less than the adhesion force, a spring-damper system joined to a wall (zero flow source) must be used. In this system, while the clot does not receive the force of minimum suction, it has a zero speed. However, when it begins its movement, the spring-damper system must be cancelled allowing its extraction.

Therefore, while the force representing the deformation of the clot is lower than the adhesion force, it will remain attached to the wall, thereby preventing any movement. To the contrary, if the force exceeds this figure the model will cause the bond imposed by the spring-damper system to be eliminated with the clot becoming free and moving in accordance with the suction pressure acting on it from the system, letting it be removed.

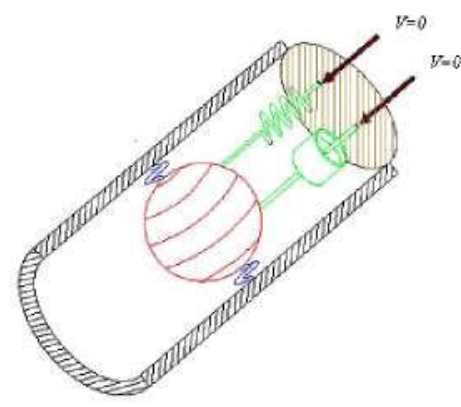

a).

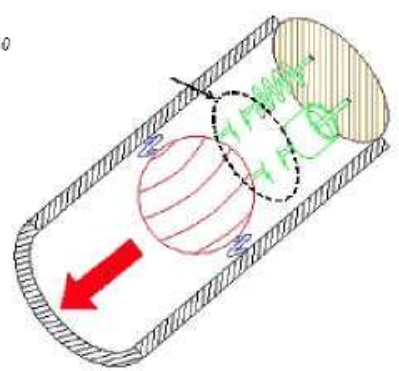

b).
Figure 3. Spring-damper system. a). F $<$ Adhe. force. b). $F \geq$ Adhe. force.

Therefore, the elimination of the spring-damper system, initially of $10^{10} \mathrm{~N} / \mathrm{m}$ and $10^{9} \mathrm{~N} \cdot \mathrm{s} / \mathrm{m}$ respectively, will be performed from the displacement of the spring representing the clot deformation, as was seen in the condition imposed on the static/dynamic friction.

In the following figure it's possible to see the full model of the complete 'GP' TAD, in which we can see the order of the different elements.

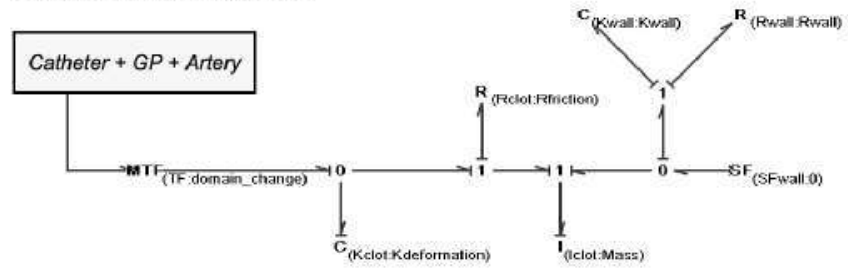

Figure 4. Modelling clot components by Bond Graph technique.

To obtain the simulation of the developed equations, Bondin (C) software [14] will allows obtaining the evolution of the characteristic parameters of the model as well as letting them be compared.

\section{RESULTS}

The aim of this simulation is to compare the extraction of a clot in the MCA considering several adhesion forces between the clot and the artery wall. This adhesion force depends on the time that the clot remains in the artery before it is extracted.

By varying the value, the movement of the clot and the time required for its extraction are measured, thereby observing how the adhesion force influences in the process. 
To carry out the model validation, the values of the parameters used in the simulation are listed in table $\mathrm{I}$, in the following page.

\begin{tabular}{l|c} 
Pressure & $0-[-30,-60] \mathrm{kPa}$ \\
\hline Blood Viscosity ( $\eta)$ & $0,0035 \mathrm{~Pa} \cdot \mathrm{s}$ \\
\hline Blood Density ( $\rho)$ & $1.060 \mathrm{~kg} / \mathrm{m}^{3}$ \\
\hline Bulk's coefficient (B) & $2,2 \cdot 10^{\circ} \mathrm{N} / \mathrm{m}$ \\
\hline Catheter length (L) & $110 \mathrm{~cm}$ \\
\hline Catheter diameter (D) & $0,001 \mathrm{~m}$ \\
\hline 'GP' length (L) & $0,020 \mathrm{~m}$ \\
\hline 'GP' diameter (D) & $0,001 \mathrm{~m}$ \\
\hline 'GP' thickness (h) & $0,0001 \mathrm{~m}$ \\
\hline Artery Young modulus (E) & $2,8 \cdot 10^{\circ} \mathrm{N} / \mathrm{m}$ \\
\hline Artery thickness (h) & $0,0001 \mathrm{~m}$ \\
\hline Artery diameter (Da) & $0,003 \mathrm{~m}$ \\
\hline Artery length (La) & $0,003 \mathrm{~m}$ \\
\hline Load loss coefficient $(\xi)$ & 0,4 \\
\hline 'GP'-artery mean diameter $\left(\mathrm{D}_{\mathrm{m}}\right)$ & $0.002 \mathrm{~m}$ \\
\hline Domain change coefficient $(\mathrm{r})$ & $141.471,06$ \\
\hline Static friction & $2,5 \cdot 10^{-6} \mathrm{~N} \cdot \mathrm{s} / \mathrm{m}$ \\
\hline Dynamic friction & $2,5 \cdot 10^{-7} \mathrm{~N} \cdot \mathrm{s} / \mathrm{m}$ \\
\hline Clot weight & $0,6-1,0 \mathrm{gr}$
\end{tabular}

Table I. Parameter values.

For each adhesion force the results show the time needed to extract two different sizes of the clot; being the pressures used the ones in the range $[-30,-60] \mathrm{kPa}$. The lengths of the clot studied are $5 \mathrm{~cm}$ and $3 \mathrm{~cm}$.

For each pressure, adhesion force and size of the clot, the necessary time to complete the extraction is picked up in the following figures.

Thanks to these results the importance of carry out the extraction of the clot as fast as it is located can be demonstrated.

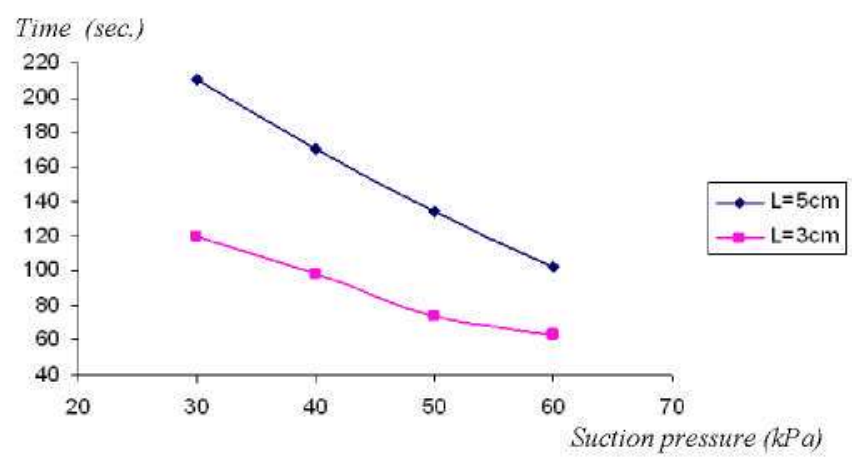

Figure 5. Adhesion force of $0,1 \mathrm{~N}$.

The first figure shows an adhesion force of $0,1 \mathrm{~N}$. It can be observed that for this force and a length of $5 \mathrm{~cm}$ of the clot, times to remove it are too high. The only value which owns to the optimum range $[60,120]$ seconds, is the value for a pressure of $-60 \mathrm{Kpa}$. It can be concluded that this value is the optimum to carry out the extraction of a length of $5 \mathrm{~cm}$.

In the same figure it can be seen that the results for a clot with a lower length $(3 \mathrm{~cm})$, each pressure can remove the clot in a lower time. It can be observed that all the values of time are in the optimum range, being possible to use any of these pressures. All the results are shown in the following figure.

If the adhesion force only reach $0,01 \mathrm{~N}$, the extraction of the $5 \mathrm{~cm}$ clot is effective by using pressures of $-40 \mathrm{kPa}$, $50 \mathrm{kPa}$ and $-60 \mathrm{kPa}$. However for a pressure of $-30 \mathrm{kPa}$ the time to carry out the extraction exceeds two minutes. For a lower length $3 \mathrm{~cm}$ clot, pressures of $-30 \mathrm{kPa}$ and $-40 \mathrm{kPa}$ are enough.

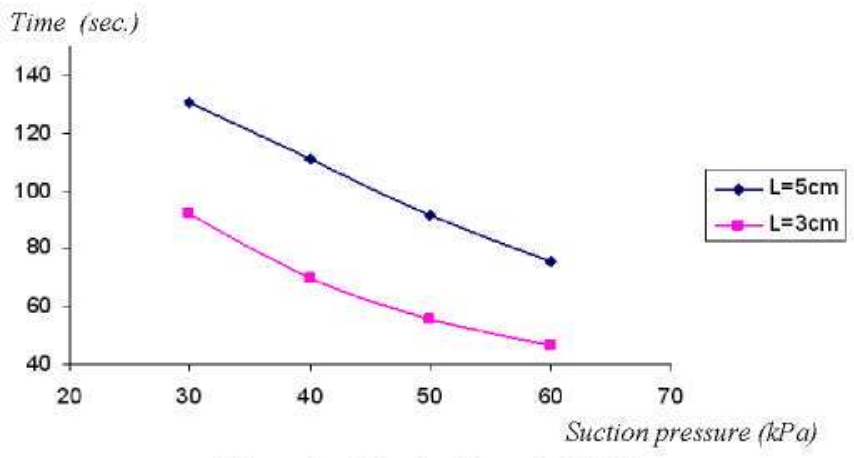

Figure 6. Adhesion force of $0,01 \mathrm{~N}$.

Finally, for an adhesion force of $0,001 \mathrm{~N}$ it can be observed that times decrease further but the differences compared with $0,01 \mathrm{~N}$ are less than that obtained if the results for 0,1 and $0,01 \mathrm{~N}$ are compared.

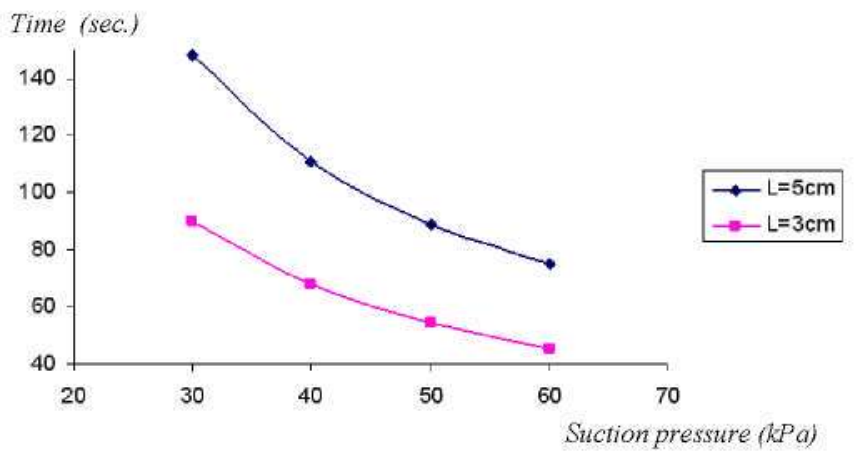

Figure 7. Adhesion force of $0,001 \mathrm{~N}$.

Like in the results of $0,01 \mathrm{~N}$, it can be concluded that for a length of $5 \mathrm{~cm}$ it is necessary to employ high pressures, $40 \mathrm{kPa},-50 \mathrm{kPa}$ or $-60 \mathrm{kPa}$. Nevertheless, for a clot length of $3 \mathrm{~cm}$, a pressure of $-30 \mathrm{kPa}$ is enough to remove the clot in a reasonable time.

\section{CONCLUSIONS}

Thanks to the development of this simulation model it has been possible to obtain an estimate of the minimum pressure required to perform the extraction for different situations of blood clot size and times of residence of the clot in the artery, and for different values of adhesion force. This study has shown that suction pressure together with the time required to complete the operation are reasonable, and are within acceptable clinical boundaries for any eventual potential use of the 'GP' TAD device on a patient, and are 
similar to experimentally obtained data. These studies may help in the optimization of the device and may assist in possible future use of this device in patients with thrombosis. although to be really effective in an future optimization of the 'GP' TAD device, ways of representing the unique design features in the 'GP' TAD device must be incorporated into any model.

For the highest adhesion force, $0,1 \mathrm{~N}$, and a length of the clot of $5 \mathrm{~cm}$. the times to remove it may be too long. The only value which produces a time in the range $[60,120]$ seconds corresponds to a pressure of $-60 \mathrm{kPa}$; and we could conclude that this level of pressure may be required to remove this size of clot. Nevertheless. for a clot of $3 \mathrm{~cm}$ all pressures modelled removed the clot in a time in the required range. So any pressure between are $-30 \mathrm{kPa}$ and $-60 \mathrm{kPa}$ can be used with a clot of $3 \mathrm{~cm}$ and an adhesion force of $0,1 \mathrm{~N}$.

If the force is decreased until $0,01 \mathrm{~N}$ and we'd like to remove a clot of $5 \mathrm{~cm}$. the pressures $-40 \mathrm{kPa}-50 \mathrm{kPa}$ and $60 \mathrm{kPa}$ give times in the optimum range; using a pressure of $30 \mathrm{kPa}$ the time increases to 130 seconds. For a length of $3 \mathrm{~cm}$. we can conclude that pressures of $-30 \mathrm{kPa}$ and $-40 \mathrm{kPa}$ are adequate, it being unnecessary to increase the pressure beyond these values.

Finally, if the force is $0,001 \mathrm{~N}$ we can appreciate that the necessary times to renrove the clot are lower but the difference when comparing the same situation for an adhesion force of $0,01 \mathrm{~N}$ is not so large as when we make the same comparison for $0,1 \mathrm{~N}$ and $0,01 \mathrm{~N}$ adhesion forces.

Moreover. analyzing the resistances in the catheter indicates that an important pressure loss takes place in the catheter joining the pump to the 'GP' device. The values obtained could possibly be used to optimize its geometry.

\section{FUTURE WORKS}

Bond Graph mathematical modelling is now being implemented to model and simulate the coronary vessels of the heart. Future analysis will involve the 'GP' TAD in proximity to both athromatous plaques and blood clots. in coronary vessels which become blocked in myocardial infarction.

\section{REFERENCES}

[1] Stroke association website. httpriwww,stroke.org.uk/document. .nn?id=330. Last accessed 30 December 2008.

[2] Guidelines for the early management of adults with ischenic stroke (2007) Stroke. 38:80-84.

[3] TAl22 Alteplase for the treatment of acute ischaemic stroke: guidanoe (2007) National Institute for clinical Excellence.

[1] Pearce G, and Perkinson ND, "Biomechanical Probe". 2006: Intenational Patent Corporate Treatise (WO2006120464) published 2006-11-16: European patent (EP1893195 (A2)) published 2008-0305: Japanese patent (JP2008639924 (T)) Published 2008-11-20: Chinese patent (CN101208049 (A)) published 2008-06-25.

[5] Pearce G, Patrick JH, Perkinson ND (2007). A new device for the treatinent of thromboembolic strokes". Jounal of Stroke and Cerebrovascular Diseases. 16(4):167-172.

[6] Pearce, G. et al, 2008. "Modelling of the 'GP' Mechanical Thrombectomy Device MTD". 10h International Conference on Computer Modeling and Sitmulation, pp. 499-502. Cambridge. UK.

[7] Pearce, G. et a]. 2009, "An Investigation of fluid flow through a modified design for the "GP" device". 11 th Intemational Conference on Computer Modelling and Simulation pp. 191-195. Cambridge. UK.

[8] Pearce, G, et al. 2008. "The design, optimisation, and testing of a new mechanical clot retrieval device for use in vascular surgery". Intemational Joumal of Engineering Simulation. Vol. 9. No. 2, pp. $10-26$.

[9] Rai. M. et al. 2009. "A Versatile Low cost Arterial Simulator", 11th Intemational Conference on Computer Modelling and Sinulation. pp. 196-199. Cambridge, UK.

[10] Kamopp. D.C. et al. 1990. "System Dynamics: A Unilied Approach", Jolu Wiley \& Sons. Inc, Second edition.

[11] Zadpoor, A.A. et al. 2005. "A bond graph approach to the modelling of fluid-solid interaction in cardiovascular system's pulsatile flow". 27th Annual International Conference of the IEEE in Medicine and Biology Society (EMBC05), Shanghai, China.

[12] Tabatabai. G.F. et al. 2005. "Spatiotemporal wavefront propagation in 3D geometric excitable hear tissue utilizing Bond Graph modelling technique". 2005 International Conference on Bond Graph Modelling and Simulation (ICBGM'2005), New Orleans, USA.

[13] Margolis, D. 1979. "Bond graph fluid line models for inclusion with dynamic systems simulations". Joumal of the Franklin Institute. Vol. 308, No. 3, pp. 255-268.

[14] Ronero, G. et al. 2009. "BONDIN: a new enginedring simulation software for $\mathrm{ODE}$ and $\mathrm{DAE}$ systems with symbolic notation based on the Bond Graph technique". 8th WSEAS Int. Conf. on Soflware engineering parallel and distributed systeuns, pp. 90-97. Cambridge. UK. 